\title{
Trends in all-cause mortality of hospitalized patients due to SARS- CoV-2 infection from a monocentric cohort in Milan (Lombardy, Italy)
}

\author{
Nicola Ughi ${ }^{1}$ (1) Davide Paolo Bernasconi ${ }^{2} \cdot$ Francesca Del Gaudio $^{3} \cdot$ Armanda Dicuonzo $^{3}$ - Alessandro Maloberti ${ }^{4,5}$. \\ Cristina Giannattasio ${ }^{4,5} \cdot$ Paolo Tarsia $^{6} \cdot$ Massimo Puoti $^{5,7}$. Francesco Scaglione ${ }^{8} \cdot$ Laura Beltrami $^{9}$. \\ Fabrizio Colombo ${ }^{9}$. Michaela Bertuzzi ${ }^{10}$. Andrea Bellone ${ }^{11}$. Antonella Adinolf ${ }^{1}$. Maria Grazia Valsecchi ${ }^{2}$. \\ Oscar Massimiliano Epis ${ }^{1} \cdot$ Claudio Rossetti ${ }^{3,12}$ on behalf of the Niguarda COVID Working Group
}

Received: 23 July 2021 / Accepted: 7 November 2021 / Published online: 4 January 2022

(c) The Author(s), under exclusive licence to Springer-Verlag GmbH Germany, part of Springer Nature 2021

\begin{abstract}
Background Robust data on case fatality rate (CFR) among inpatients with COVID-19 are still lacking, and the role of patient characteristics in in-hospital deaths remains under-investigated. This study quantified the overall CFR and described its trend in a cohort of hospitalized patients with SARS-CoV-2 in Italy. Admission to ICU, death, or discharge were the secondary outcomes. Methods This retrospective study is based on administrative health data and electronic case records of inpatients consecutively admitted to Niguarda Hospital between 21 February and 8 November 2020.

Results An overall CFR of $18 \%$ was observed. CFR was significantly reduced during the second wave of contagion (1 June to 30 September, $16 \%$ ) compared with the first wave (21 February to 31 May, $21 \% p=0.015$ ). Such reduction was mainly observed among male inpatients between 40 and 80 years with limited comorbidities. Admission to ICU was associated with a high risk of mortality in both waves. The incidence of severe disease and the need for ICU admission were lower in the second wave.

Conclusion CFR in SARS-CoV-2 inpatients was demonstrated to decrease over time. This reduction may partly reflect the changes in hospital strategy and clinical practice. The reasons for this improvement should be further investigated to plan an exit strategy in case of future outbreaks.
\end{abstract}

\section{Key messages}

What is already known on this topic

Before the advent of anti-COVID-19 vaccines, a multi-wave pattern of contagion was observed, and this trend conditioned the inpatient case fatality rate (CFR), which varied over time accordingly to the waves of contagion.

Only preliminary results on the in-hospital mortality trend are available, along with a partial analysis of its determinants. Consequently, robust data on CFR among inpatients with SARS-CoV-2 infection are still lacking, and the role of patient characteristics in in-hospital deaths remains under-investigated.

What this study adds

This study shows that the in-hospital mortality in patients with SARS-CoV-2 infection decreases over time.

Such reduction was mainly observed among male inpatients between 40 and 80 years with limited comorbidities. Admission to ICU was invariably associated with a high risk of mortality during the whole study period (21 February to 8 November 2020), but the incidence of severe disease and the need for ICU admission were lower in the second wave of contagions (1 October to 8 November 2020). This reduction may partly reflect the impact of changes in hospital strategy and clinical practice. The reasons for this improvement should be further investigated to inform the response to future outbreaks and to plan exit strategy by prioritizing high-risk populations.

Keywords SARS-CoV-2 infection $\cdot$ COVID-19 $\cdot$ Case fatality rate $\cdot$ Inpatients with COVID-19 $\cdot$ Trend of case fatality rate

\section{Introduction}

Nicola Ughi and Davide Bernasconi contributed equally to the manuscript and shared the first authorship.

Extended author information available on the last page of the article
By the end of 2019, the severe acute respiratory syndrome coronavirus 2 (SARS-CoV-2) had spread as coronavirus disease 2019 (COVID-19) (Hu et al. 2021). Since the WHO 
announced the COVID-19 outbreak to be a pandemic (12 March 2020) (WHO 2020), a burden imposed upon global health and excess mortality was observed, concerning both COVID-19 and the delayed care due to overwhelmed healthcare systems (Woolf et al. 2021; Reese 2019).

Before the advent of anti-COVID-19 vaccines, a multi-wave pattern of contagion was observed globally, and this reflected on national health policies, which had to face up different levels of hospital care burden (Cacciapaglia et al. 2021). In addition, this multi-wave pattern also conditioned the inpatient case fatality rate (CFR), which varied over time according to the wave of contagion (Madahar et al. 2021; Horwitz et al. 2021; Saito et al. 2021).

To better characterize this phenomenon, the host characteristics were substantially investigated, and age, gender, and the presence of comorbidities were considered as major factors related to infection mortality, from the first months of the pandemic onset (Li et al. 2021). The impact of such determinants on in-hospital deaths seems to have been confirmed through the pandemic waves (Navaratnam et al. 2021), but this issue remains under-investigated.

Italy was among the first countries that faced the COVID19 health emergency; the first case of pneumonia due to SARS-CoV-2 was diagnosed in northern Italy on 20 February 2020 (Onder et al. 2020). Since then, a bimodal pattern of the pandemic spread was observed throughout 2020. The first wave subsided by the end of May (Alicandro et al. 2020) and a second wave was registered in the second half of 2020. Even if preliminary results on the in-hospital mortality trend in northern Italy are available (Borghesi et al. 2021), along with a partial analysis about its determinants (Navaratnam et al. 2021), robust data on CFR among inpatients with SARS-CoV-2 infection are still lacking and the role of patient characteristics in in-hospital deaths remains under-investigated.

This study aimed to assess the overall CFR and to describe its trend during 2020 in a monocentric cohort of hospitalized patients with a diagnosis of SARS-CoV-2 infection in northern Italy. Furthermore, admission to ICU, death, or discharge from the moment of admission have been considered as secondary outcomes.

\section{Patients and methods}

\section{Study design, setting, and participants}

This is a retrospective cohort study based on the review of administrative health data and electronic case records of the inpatients who were consecutively admitted to Niguarda Hospital between 21 February and 8 November 2020. Niguarda is one of the largest General Hospital in the north of Milan within a Metropolitan Area of 3,279,944 inhabitants (January 2020) and hosts all the medical and surgical disciplines for adults and children, including a 24-hour Emergency Department with 96,588 visits and 32,612 hospital admissions covering every intensity of care in 2019. In this hospital context, all inpatients were eligible if diagnosed with SARS-Cov-2 infection, certified by the positivity of nasopharyngeal swab to SARS-CoV-2 genome, regardless of the presence of the respiratory disease. Most of the swab tests were processed at Niguarda Hospital, and a documented result of test positivity from other health facilities was not a reason for exclusion. Inpatients who developed COVID-19 symptoms and/or tested positive for the SARS-CoV-2 genome after 48 hours from the admission were excluded, as their infections could be considered to be possibly hospital-acquired. All the included patients were followed up from the first day of either elective or urgent admission in the hospital until the day of discharge, death, or 17 January 2021.

This study conformed to the Helsinki Declaration and was approved by the ethics committee for Milano Area 3 (register number 249-13052020). Informed consent was provided by the participants.

\section{Outcome measurements}

The overall CFR was the main outcome, and it was calculated as the proportion of observed in-hospital deaths (all-cause mortality) over the total number of included inpatients with a diagnosis of SARS-CoV-2 infection in the period of observation. Then, time-specific CFRs were calculated and to this end, the study period was divided into the following three periods, consistent with the trends of hospital admissions due to SARS-CoV-2 infection: 1) first wave: from 21 February to 31 May 2020,2) intermediate phase: between 1 June and 30 September 2020 , and 3) second wave: from 1 October to 8 November 2020. CFRs were compared between the first- and second-wave patient groups.

Finally, specific CFRs were estimated with respect to the following subgroups, according to the first and second wave time intervals: gender, age levels in terms of decades (between $<20$ years and $\geq 90$ years), admission to an intensive care unit (ICU), and comorbidity burden defined under the Charlson comorbidity index (Charlson et al. 1987) and stratified in five categories (from score 0 to $\geq 5$ ). Moreover, CFR was also investigated by subgrouping patients based on disease severity - i.e., whether pulmonary involvement was detected.

Secondary outcomes were defined from the time of admission to the following events: admission to ICU, death, or discharge. Both single and composite outcomes reflecting complex definitions of in-hospital SARS-CoV-2 infection 
prognosis were considered to investigate the secondary endpoints.

\section{Statistical analysis}

The distribution of patients' characteristics at admission was described using numbers and frequencies for categorical variables, median, and interquartile range (IQR) or mean and standard deviation (SD) for continuous variables. These characteristics were compared between the first- and secondwave patient groups using the Chi-square test, Mann-Whitney test or $t$-test, as appropriate. A description of the patients hospitalized during the intermediate phase was also performed. Point estimates of overall and subgroup-specific CRFs and their $95 \%$ confidence intervals (CI), according to the Wald method, were calculated.

To analyze the incidence of all-cause mortality and admission to ICU, a time-to-event analysis was performed accounting for discharge (treated as a competing risk) and the presence of patients that were still in hospital at the moment of data extraction (treated as censored observations). The Aalen-Johansen estimator was used to estimate the crude incidence of the two following competing events within the two waves: admission to ICU or death before ICU (composite outcome) and discharge before ICU. The curves were compared between the two waves using the Gray test (Gray 1988). A similar analysis was performed on the three following competing endpoints: admission to ICU, death before ICU, and discharge before ICU. An additional analysis was conducted for the two following unique competing events: in-hospital death and discharge.

To assess the association of patients' characteristics (age, gender, Charlson comorbidity index) at baseline with the afore-mentioned outcomes, univariate cause-specific Cox models were fitted, separately for each wave. Finally, a multiple cause-specific Cox model jointly on the populations of both waves was fitted to compare the hazard of each event between the two waves by adjusting for age, gender and Charlson comorbidity index. All these analyses were based on complete cases.

All the analyses were performed using R 4.0.3 (R Core Team, 2020. R: A language and environment for statistical computing. R Foundation for Statistical Computing, Vienna, Austria).

\section{Results}

\section{Study population}

In the study period, 1859 individuals admitted to Niguarda Hospital presented with the SARS-CoV-2 infection. In total, 74 patients who developed COVID-19 symptoms and/or tested positive for SARS-Cov-2 after 48 hours from admission were excluded (Supplementary Fig. S1). The remaining 1785 patients were classified according to the period of admission: first wave $(920,52 \%)$, intermediate phase (133, $7 \%)$, or second wave $(732,41 \%)$. A comparison of the characteristics of the patients between the first and second waves is shown in Table 1.

The median time between the onset of symptoms and hospital admission was significantly shorter for the second wave (median [IQR]: 5 days [3-8] vs 6 [3-9], $p=0.016$ ).

Patients of the second-wave group were significantly older (median [IQR]: 66 years [54.37-78.82] vs 64 [51.74-77.07], $p=0.020$ ) and with more comorbidities (Charlson comorbidity index mean [SD]: 4 [3] vs 3 [3], $p<0.001$ ) compared with the first-wave group. In particular, more patients with a history of myocardial infarction $(13 \%$ vs $8 \%, p=0.001)$, cerebrovascular accident or transient ischemic attack (12\% vs $8 \%, p=0.004)$ and solid tumors $(15 \%$ vs $10 \%, p=0.006)$ were observed in the second-wave group.

Nevertheless, admissions to ICU were significantly higher in the first-wave group ( $18 \%$ vs $9 \%, p<0.001)$.

Patients admitted during the intermediate phase were markedly different from those of the two waves, as shown in Supplementary Table 1.

The trend of admissions and outcomes (deaths and discharges), in terms of absolute numbers, occurred in each week from 21 February to 31 December 2020 is reported in Supplementary Fig. S2. The peak of admissions per week during the second wave is higher than in the first wave (218 vs 171) but with a similar peak of deaths (37 vs 34). The peaks of discharges occurring later than 2 weeks after admissions are also evident in both waves.

\section{Overall CFR}

The overall CFR in the study period was $18.5 \%$ (95\% CI: 16.7-20.3; the number of deaths/number of cases: $330 / 1785)$.

Over a median of 14 [IQR 8-23] days of follow-up for patients of the first wave and 11 [IQR 7-17] days for patients of the second wave, 197 and 121 fatalities were observed respectively (number of cases: 920 in the first wave, 732 in the second wave), with a CFR (95\% CI) significantly lower in the second-wave group of patients: $21.4 \%(18.8-24.1)$ vs $16.5 \%$ (13.8-19.2), $p=0.015$.

CFR of the intermediate phase was 9\% (95\% CI: 4.2-13.9), considering 12 deaths out of 133 patients.

A comparison of the CFR between the two waves calculated in population subgroups (gender, age categories, admission to ICU, Charlson comorbidity index) is shown in Fig. 1. The CFR was lower in the second wave than in the first for all subgroups but especially within males ( $17 \%$ vs $24 \%$ ), age $\geq 60$ to $<70$ ( $11 \%$ vs $21 \%), \geq 70$ to $<80$ 
Table 1 Comparison of patients' characteristics at admission between waves

\begin{tabular}{|c|c|c|c|}
\hline Variables & Wave $1(n=920)$ & Wave $2(n=732)$ & $P$-value* \\
\hline Gender (male), $n(\%)$ & $575(62.5)$ & $490(66.9)$ & 0.069 \\
\hline Age (years), median (IQR) & $64.06(51.74-77.07)$ & $66.44(54.37-78.82)$ & 0.020 \\
\hline Age (years), $n(\%)$ & & & 0.060 \\
\hline$<20$ & $27(2.9)$ & $27(3.7)$ & \\
\hline$\geq 20$ to $<30$ & $52(5.7)$ & $28(3.8)$ & \\
\hline$\geq 30$ to $<40$ & $111(12.1)$ & $62(8.5)$ & \\
\hline$\geq 40$ to $<50$ & $197(21.4)$ & $136(18.6)$ & \\
\hline$\geq 50$ to $<60$ & $154(16.7)$ & $149(20.4)$ & \\
\hline$\geq 60$ to $<70$ & $192(20.9)$ & $162(22.1)$ & \\
\hline$\geq 70$ to $<80$ & $148(16.1)$ & $132(18.0)$ & \\
\hline$\geq 80$ to $<90$ & $14(1.5)$ & $11(1.5)$ & \\
\hline$\geq 90$ & $25(2.7)$ & $25(3.4)$ & \\
\hline Current pregnancy, $n(\%)$ & $3(0.3)$ & $4(0.5)$ & 0.761 \\
\hline Days from first symptoms to admission, median (IQR) & $6(3-9)$ & $5(3-8)$ & 0.016 \\
\hline Severe disease (any pulmonary involvement), $n(\%)$ & $789(85.8)$ & $648(88.5)$ & 0.113 \\
\hline Admission to ICU & $169(18.4)$ & $65(8.9)$ & $<0.001$ \\
\hline \multicolumn{4}{|l|}{ History of: } \\
\hline Asthma, $n(\%)$ & $33(4.8)$ & $23(3.1)$ & 0.146 \\
\hline Myocardial infarction, $n(\%)$ & $72(7.8)$ & $94(12.9)$ & 0.001 \\
\hline Congestive heart failure, $n(\%)$ & $55(6.0)$ & $33(4.5)$ & 0.224 \\
\hline Peripheral vascular disease, $n(\%)$ & $52(5.7)$ & $53(7.3)$ & 0.225 \\
\hline Cerebrovascular accident or transient ischemic attack, $n(\%)$ & $71(7.7)$ & $88(12.0)$ & 0.004 \\
\hline Dementia, $n(\%)$ & $62(6.8)$ & $48(6.6)$ & 0.954 \\
\hline Chronic obstructive pulmonary disease, $n(\%)$ & $58(6.3)$ & $64(8.8)$ & 0.074 \\
\hline Peptic ulcer disease, $n(\%)$ & $17(1.8)$ & $20(2.7)$ & 0.298 \\
\hline Hemiplegia, $n(\%)$ & $16(1.7)$ & $16(2.2)$ & 0.643 \\
\hline Liver disease, $n(\%)$ & $22(2.4)$ & $25(3.4)$ & 0.277 \\
\hline Moderate to severe chronic kidney disease, $n(\%)$ & $81(8.8)$ & $67(9.2)$ & 0.877 \\
\hline Solid tumor, $n(\%)$ & $95(10.3)$ & $109(14.9)$ & 0.006 \\
\hline AIDS, $n(\%)$ & $2(0.2)$ & $6(0.8)$ & 0.163 \\
\hline Diabetes mellitus, $n(\%)$ & $146(15.9)$ & $128(17.5)$ & 0.427 \\
\hline Leukemia, $n(\%)$ & $12(1.3)$ & $5(0.7)$ & 0.318 \\
\hline Lymphoma, n (\%) & $18(2.0)$ & $11(1.5)$ & 0.609 \\
\hline Charlson comorbidity Iindex, 0-37 score, mean (SD) & $3(3)$ & $4(3)$ & $<0.001$ \\
\hline Charlson comorbidity index, estimated 10 -year survival (\%), mean (SD) & $62(39)$ & $57(39)$ & 0.003 \\
\hline Charlson comorbidity index, $n(\%)$ & & & 0.001 \\
\hline Score 0 & $263(28.6)$ & $253(34.6)$ & \\
\hline Score 1 & $190(20.7)$ & $92(12.6)$ & \\
\hline Score 2 & $130(14.1)$ & $95(13.0)$ & \\
\hline Score 3 & $124(13.5)$ & $102(14.0)$ & \\
\hline Score 4 & $105(11.4)$ & $96(13.1)$ & \\
\hline Score $\geq 5$ & $107(11.6)$ & $93(12.7)$ & \\
\hline
\end{tabular}

*Chi-square test for categorical, Mann-Whitney test or $t$-test for numerical variables; ICU, intensive care unit; IQR, interquartile range; sd, standard deviation. $P$-values reported in bold are statistically significant

(22\% vs $33 \%), \geq 90(60 \%$ vs $40 \%)$, patients not admitted to ICU (14\% vs $17 \%$ ) and patients with Charlson comorbidity index [2 (4\% vs 16\%) and $3(9 \%$ vs $29 \%)$ ]. Moreover, the decrease in CFR in the second wave was more evident within patients with mild disease ( $2 \%$ vs $13 \%$ ) than with severe disease (18\% vs 23\%), as shown in Supplementary Table 2. 
Fig. 1 Comparison of case fatality rate (CFR) between the two waves in subgroups: gender (A), age (B), admission to intensive care unit $(\mathbf{C})$, Charlson Comorbidity Index (D). Bars are $95 \%$ CIs.
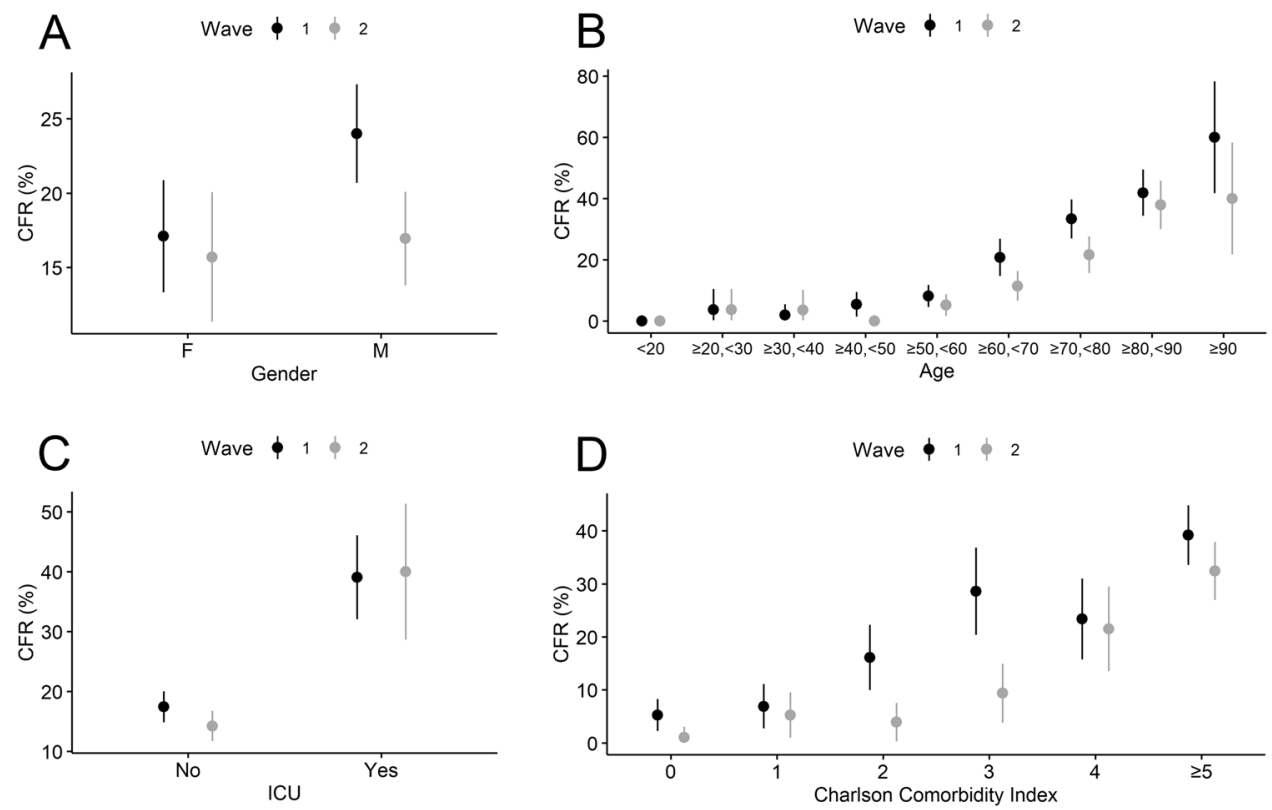

\section{Secondary outcomes}

The crude cumulative incidence as estimated by the Aalen-Johansen estimator of the competing risks (ICU or death without ICU and discharge without ICU) in the two waves are depicted in Fig. 2. At 30 days after admission, the estimated cumulative incidence of ICU or death without ICU (Fig. 2A) was 33\% (95\% CI: $30-36.2$ ) and $22 \%$ $(19-25.3)$ respectively for waves 1 and $2(p<0.001)$. The estimated incidence of discharge without ICU (Fig. 2B) was $54 \%(50.1-57)$ in wave 1 and 65\% (61.6-69.3) in wave 2 $(p<0.001)$. At 30 days we also observed an incidence of only ICU (Fig. 2C) of 19\% (16-21.1) vs 9\% (7-11.3), $p<0.001$, and an incidence of death with or without ICU (Fig. 2C) of $21 \%(18.5-24)$ vs $17 \%$ (13.6-19.6), $p=0.109$ respectively for wave 1 versus wave 2 .

According to the Cox univariate analysis, older age and higher Charlson comorbidity index were significantly associated with a higher hazard of ICU or death and a lower hazard of discharge in both waves, while male gender was a stronger predictor of worse outcome during the first than during the second wave (Table 2).

Multiple Cox model analysis adjusting for age, gender, and Charlson comorbidity index indicates that the hazard of ICU or death during wave 2 was significantly lower than during wave 1 (HR with 95\% CI: 0.65, 0.54-0.79). The hazard of discharge without ICU was significantly higher during wave $2(1.46,1.28-1.67)$ than the hazard of discharge with or without ICU $(1.50,1.32-1.71)$ while the hazard of death with or without ICU was not significantly different $(0.83,0.66-1.04)$, as shown in Supplementary Table 3.

\section{Discussion}

Between February and November 2020, a CFR of $18 \%$ (95\% CI: 16.7-20.3) was observed among patients admitted to Niguarda Hospital with SARS-CoV-2 infection; this was significantly reduced during the second wave $(16.5 \%$, 95\% CI: $13.8-19.2)$ compared to the first one $(21.4 \%, 95 \%$ CI: $18.8-24.1 ; p=0.015)$. Such reduction was mainly observed among male inpatients who were over 40 years and under 80 years and with a limited burden of comorbidities, while the CFR was steadily low in young females with no comorbidities and high among elderly in-patients with multiple comorbidities. Admission to ICU was invariably associated with a high risk of mortality in both waves, but the incidence of severe disease (i.e., requiring intensive care or leading to death before ICU admission) and the need for ICU admission was lower in the second wave compared to the first one.

In-hospital mortality in patients with SARS-CoV-2 infection was variably high across several international studies, and consistent with our findings. The estimate of CFR during the first months of the outbreak in 2020 was 17\% (95\% CI: $12.7-22.7, n=13,398$ ) in a meta-analysis on 33 studies between January and April 2020, and mortality was lower in general inpatients $(11 \%, 95 \%$ CI: 7.7-16.9) compared with critically ill patients (40\%, 95\% CI: 31.2-50.6) (Macedo et al. 2021). However, these reports unraveled a degree of heterogeneity with geographical differences, and mortality up to $31 \%$ was reported, as in the case of 522,167 patients hospitalized with SARS-CoV-2 infection in Brazil by December 2020 (Castro et al. 2021). Further to the issues related to CFR calculation methods, such diversity of results might reflect 
Fig. 2 Crude incidence (AalenJohansen estimates) of admission to intensive care unit or death before intensive care unit (A), discharge before intensive care unit $(\mathbf{B})$, admission to intensive care unit $(\mathbf{C})$, and death in hospital (D)
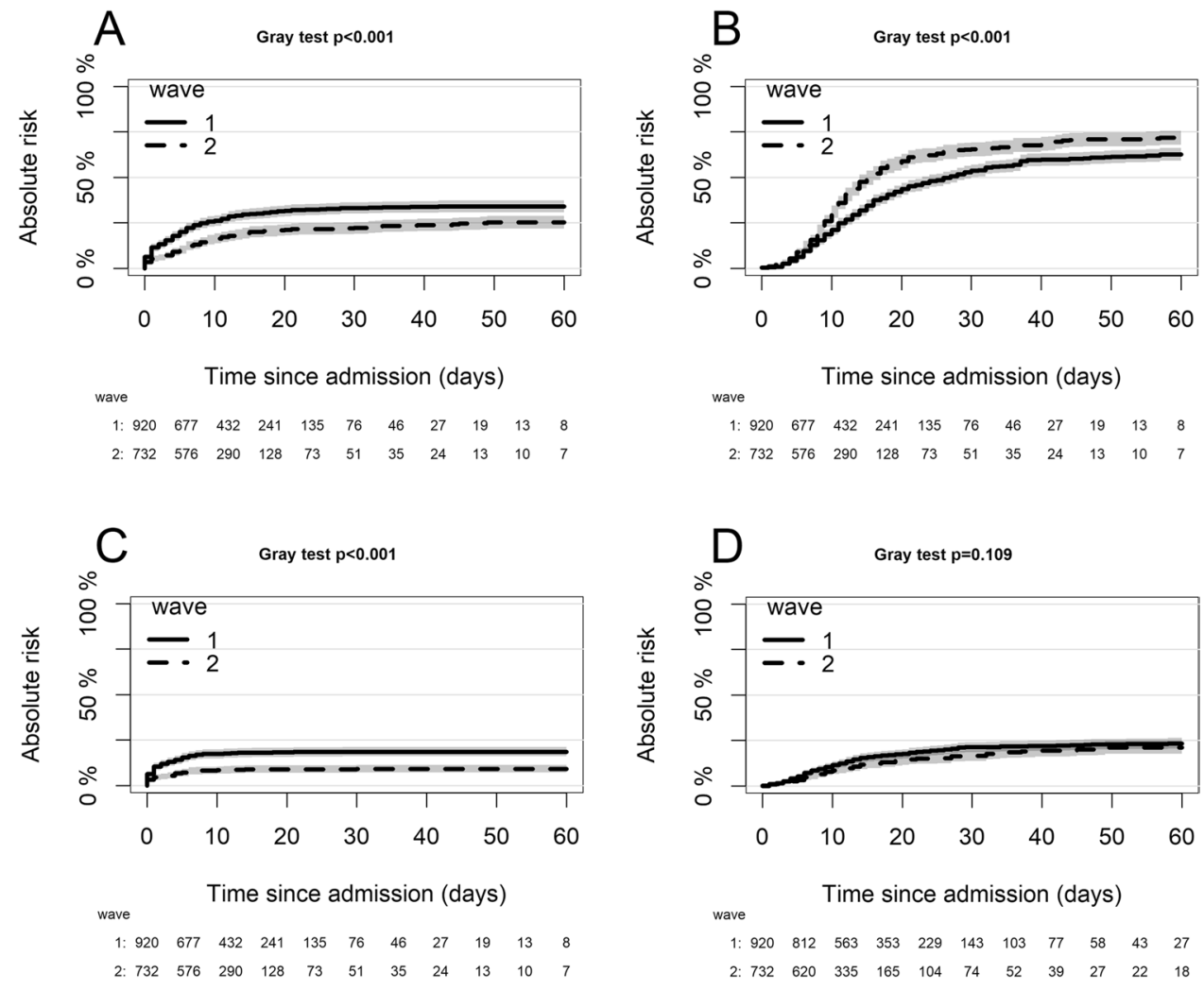

the SARS-CoV-2 virulence on different populations, as well as the local capacity of the national healthcare systems.

A worldwide change in the trend of in-hospital mortality of SARS-CoV-2 infection over the year 2020 was described by several studies —from Japan (Saito et al. 2021), USA (Horwitz et al. 2021; Roth et al. 2021; Finelli et al. 2021), UK (Navaratnam et al. 2021; Docherty et al. 2021) and Spain (Domingo et al. 2021; Garcia-Vidal et al. 2021) and a drop in in-hospital mortality rates were also confirmed in our Italian cohort. It is not known if this change may be primarily attributed to the accrual of clinical knowledge on pharmacological treatment, such as the optimization of the management of corticosteroids (Pulakurthi et al. 2021) or to the training of healthcare workers to rapidly provide support during hospital overflow. Conversely, disease mortality was also observed to be unchanged over time, as in the case of Brazil (Castro et al. 2021), and additional factors such as peculiar health policies of infection prevention and inequities in healthcare systems might have a role.

An older age ( $>60$ years) and the presence of comorbidities were found to be independent predictors of severe outcomes in hospitalized patients with SARS-CoV-2 infection, in line with other case studies from Spain and Italy (Corradini et al. 2021; Moreno-Torres et al. 2021). The role of the male gender is controversial. Sex differences in COVID-19 case fatality were observed in the first half of 2020, and male gender seemed to be associated with poor outcomes (Li et al. 2021). However, this association was inconsistently reported across studies (Dehingia and Raj 2021). In our results, a significant association was observed with regard to the first wave (HR 1.40, 95\% CI: $1.03-1.89$, female as reference), and yet not in the second one (HR 0.81, 95\% CI: 0.55-1.19). This apparent gender gap was hypothesized to reflect other factors such as the access to health services and its representation in hospital settings, rather than biological differences in response to SARS-CoV-2 pathology (Dehingia and Raj 2021).

This study had several limitations. First, the monocentric nature of data source and analysis may reflect regional and local health policies and might not be generalized to all the hospital settings on a country level. Secondly, all-cause death and all cases requiring ICU were considered as outcomes independently of the association with the severity of COVID-19, and the inclusion of SARS-CoV-2 patients without a severe disease might over- or under-estimate CFR and outcome predictors. Finally, several major predictors, such as in-hospital treatment, concurrent complications due to hospitalization, social determinants and its proxies, such as ethnicity, were not considered in the analysis, and the effect size of age, sex, and comorbidities may reflect other features, such as social status.

In conclusion, the observed in-hospital mortality in patients with SARS-CoV-2 infection was shown to decrease over time, and this reduction may partly reflect 
Table 2 Univariate cause-specific Cox models hazard ratios with 95\% confidence interval to assess the association of patients' characteristics at baseline with each event, separately on each wave.

\begin{tabular}{|c|c|c|c|c|}
\hline \multirow[t]{2}{*}{ Factors } & \multicolumn{2}{|c|}{ ICU or death without ICU, HR $(95 \%$ CI $)$} & \multicolumn{2}{|c|}{ Discharge without ICU, HR (95\% CI) } \\
\hline & $\begin{array}{l}\text { Wave } 1 \\
n=920 \\
N \text { events }=300\end{array}$ & $\begin{array}{l}\text { Wave } 2 \\
n=732 \\
N \text { events }=160\end{array}$ & $\begin{array}{l}\text { Wave } 1 \\
n=920 \\
N \text { events }=489\end{array}$ & $\begin{array}{l}\text { Wave } 2 \\
n=732 \\
N \text { events }=429\end{array}$ \\
\hline Age (per 10-year increment) & $1.171(1.092-1.256)$ & $1.293(1.156-1.446)$ & $0.790(0.755-0.826)$ & $0.756(0.719-0.800)$ \\
\hline $\begin{array}{l}\text { Gender, } \\
\text { female as reference }\end{array}$ & $1.625(1.265-2.088)$ & $1.128(0.799-1.594)$ & $0.962(0.804-1.153)$ & $0.830(0.677-1.016)$ \\
\hline \multicolumn{5}{|l|}{$\begin{array}{l}\text { Charlson comorbidity index, } \\
\text { Score } 0 \text { as reference }\end{array}$} \\
\hline Score 1 & $1.695(1.078-2.667)$ & $1.431(0.593-3.454)$ & $1.091(0.820-1.452)$ & $0.734(0.530-1.017)$ \\
\hline Score 2 & $1.898(1.222-2.950)$ & $1.310(0.549-3.125)$ & $0.716(0.531-0.964)$ & $0.550(0.397-0.761)$ \\
\hline Score 3 & $1.736(1.100-2.740)$ & $1.532(0.655-3.585)$ & $0.608(0.443-0.834)$ & $0.469(0.334-0.659)$ \\
\hline Score 4 & $1.372(0.855-2.203)$ & $1.783(0.791-4.021)$ & $0.497(0.366-0.676)$ & $0.243(0.168-0.353)$ \\
\hline Score $\geq 5$ & $1.737(1.190-2.535)$ & $2.550(1.230-5.288)$ & $0.302(0.232-0.393)$ & $0.201(0.148-0.272)$ \\
\hline \multirow[t]{2}{*}{ Factors } & Death, HR (95\% CI) & & Discharge, HR (95\% C & \\
\hline & $\begin{array}{l}\text { Wave } 1 \\
n=920 \\
N \text { events }=197\end{array}$ & $\begin{array}{l}\text { Wave } 2 \\
n=732 \\
N \text { events }=121\end{array}$ & $\begin{array}{l}\text { Wave } 1 \\
n=920 \\
N \text { events }=555\end{array}$ & $\begin{array}{l}\text { Wave } 2 \\
n=732 \\
N \text { events }=443\end{array}$ \\
\hline Age (per 10-year increment) & $1.689(1.515-1.883)$ & $1.969(1.669-2.322)$ & $0.808(0.770-0.848)$ & $0.778(0.738-0.820)$ \\
\hline Gender (female as reference) & $1.396(1.028-1.894)$ & $0.812(0.552-1.195)$ & $0.899(0.758-1.065)$ & $0.789(0.646-0.964)$ \\
\hline \multicolumn{5}{|l|}{$\begin{array}{l}\text { Charlson comorbidity index, } \\
\text { Score } 0 \text { as reference }\end{array}$} \\
\hline Score 1 & $1.012(0.448-2.714)$ & $3.758(0.439-32.18)$ & $0.789(0.609-1.021)$ & $0.671(0.487-0.923)$ \\
\hline Score 2 & $2.287(1.069-4.897)$ & $2.619(0.293-23.44)$ & $0.551(0.418-0.728)$ & $0.573(0.418-0.787)$ \\
\hline Score 3 & $4.574(2.233-9.370)$ & $6.341(0.803-50.08)$ & $0.627(0.465-0.844)$ & $0.513(0.368-0.715)$ \\
\hline Score 4 & $3.689(1.771-7.684)$ & $12.870(1.725-96.03)$ & $0.641(0.481-0.854)$ & $0.345(0.241-0.494)$ \\
\hline Score $\geq 5$ & $6.535(3.413-12.515)$ & $18.448(2.564-132.72)$ & $0.416(0.323-0.536)$ & $0.279(0.208-0.373)$ \\
\hline
\end{tabular}

HR, hazard ratio; CI, confidence interval

the impact of changes in hospital strategy and clinical practice. The reasons for this improvement should be further investigated to inform the response to future outbreaks and to plan exit strategy by prioritizing high-risk populations.

Supplementary Information The online version contains supplementary material available at https://doi.org/10.1007/s10389-021-01675-y.

Acknowledgments Editorial assistance was provided by Simonetta Papa, PhD, and Aashni Shah (Polistudium SRL, Milan, Italy).

Authors' contributions Study conception and design: Nicola Ughi, Davide Paolo Bernasconi, Maria Grazia Valsecchi, Oscar Massimiliano Epis, Claudio Rossetti. Data collection: Nicola Ughi, Francesca Del Gaudio, Armanda Dicuonzo, Laura Beltrami, Michaela Bertuzzi. Data interpretation: all. Statistical analysis: Davide Paolo Bernasconi, Maria Grazia Valsecchi, Nicola Ughi Manuscript drafting: Nicola Ughi, Davide Paolo Bernasconi. Manuscript editing and approval to submit: all.

Funding There was no explicit funding for the development of this work.
Availability of data and material Data may be made available upon reasonable request.

Code availability Not applicable

\section{Declarations}

Conflicts of interest None of the authors declared conflict of interests.

Ethics approval This study was approved by the ethics committee Milano Area 3 (register number 249-13052020).

Consent to participate All the participants signed an informed consent form.

Consent for publication Not required as this manuscript doesn't include details, images, or videos related to the participants

\section{References}

Alicandro G, Remuzzi G, La Vecchia C (2020) Italy's first wave of the COVID-19 pandemic has ended: no excess mortality in May. Lancet 396(10253):e27-e28. https://doi.org/10.1016/S0140-6736(20) 31865-1 
Borghesi A, Golemi S, Carapella N, Zigliani A, Farina D, Maroldi R (2021) Lombardy, Northern Italy: COVID-19 second wave less severe and deadly than the first? A preliminary investigation. Infect Dis (Lond) 53(5):370-375. https://doi.org/10.1080/23744235.2021. 1884745

Cacciapaglia G, Cot C, Sannino F (2021) Multiwave pandemic dynamics explained: how to tame the next wave of infectious diseases. Sci Rep. 11(1):6638. https://doi.org/10.1038/s41598-021-85875-2

Castro MC, Gurzenda S, Macário EM, França GVA (2021) Characteristics, outcomes and risk factors for mortality of 522,167 patients hospitalised with COVID-19 in Brazil: a retrospective cohort study. BMJ Open 11(5):e049089. https://doi.org/10.1136/bmjop en-2021-049089

Charlson ME, Pompei P, Ales KL, MacKenzie CR (1987) A new method of classifying prognostic comorbidity in longitudinal studies: development and validation. J Chronic Dis 40(5):373-383. https://doi.org/ 10.1016/0021-9681(87)90171-8

Corradini E, Ventura P, Ageno W et al (2021) Clinical factors associated with death in 3044 COVID-19 patients managed in internal medicine wards in Italy: results from the SIMI-COVID-19 study of the Italian Society of Internal Medicine (SIMI). Intern Emerg Med 16(4):1005-1015. https://doi.org/10.1007/s11739-021-02742-8

Dehingia N, Raj A (2021) Sex differences in COVID-19 case fatality: do we know enough? Lancet Glob Health 9(1):e14-e15. https://doi.org/ 10.1016/S2214-109X(20)30464-2

Docherty AB, Mulholland RH, Lone NI et al (2021) Changes in inhospital mortality in the first wave of COVID-19: a multicentre prospective observational cohort study using the WHO Clinical Characterisation Protocol UK. Lancet Respir Med 9(7):773-785. https://doi.org/10.1016/S2213-2600(21)00175-2

Domingo P, Pomar V, Mur I, Castellví I, Corominas H, de Benito N (2021) Not all COVID-19 pandemic waves are alike. Clin Microbiol Infect 27(7):1040.e7-1040.e10. https://doi.org/10.1016/j.cmi. 2021.04.005

Finelli L, Gupta V, Petigara T, Yu K, Bauer KA, Puzniak LA (2021) Mortality among US patients hospitalized with SARS-CoV-2 infection in 2020. JAMA Netw Open 4(4):e216556. https://doi.org/10.1001/ jamanetworkopen.2021.6556

Garcia-Vidal C, Cózar-Llistó A, Meira F et al (2021) Trends in mortality of hospitalised COVID-19 patients: a single centre observational cohort study from Spain. Lancet Reg Health Eur 3:100041. https:// doi.org/10.1016/j.lanepe.2021.100041

Gray RJ (1988) A class of K-sample tests for comparing the cumulative incidence of a competing risk. Ann Stat 16(3):1141-1154

Horwitz LI, Jones SA, Cerfolio RJ, et al. (2021) Trends in COVID-19 risk-adjusted mortality rates. J Hosp Med 16(2):90-92. https://doi. org/10.12788/jhm.3552.P

Hu B, Guo H, Zhou P, Shi ZL (2021) Characteristics of SARS-CoV-2 and COVID-19. Nat Rev Microbiol 19(3):141-154. https://www.nature. com/articles/s41579-020-00459-7.
Li Y, Ashcroft T, Chung A, Dighero I, Dozier M, Horne M, McSwiggan E, Shamsuddin A, Nair H (2021) Risk factors for poor outcomes in hospitalised COVID-19 patients: A systematic review and metaanalysis. J Glob Health 11:10001. https://doi.org/10.7189/jogh.11. 10001

Macedo A, Gonçalves N, Febra C (2021) COVID-19 fatality rates in hospitalized patients: systematic review and meta-analysis. Ann Epidemiol 57:14-21. https://doi.org/10.1016/j.annepidem.2021.02.012

Madahar P, Wunsch H, Jha P, Slutsky AS, Brodie D (2021) Trends in COVID-19-related in-hospital mortality: lessons learned from nationwide samples. Lancet Respir Med 9(4):322-324. https://doi. org/10.1016/S2213-2600(21)00080-1

Moreno-Torres V, de la Fuente S, Mills P et al (2021) Major determinants of death in patients hospitalized with COVID-19 during the first epidemic wave in Madrid, Spain. Medicine (Baltimore) 100(16):e25634. https://doi.org/10.1097/MD.0000000000025634

Navaratnam AV, Gray WK, Day J, Wendon J, Briggs TWR (2021) Patient factors and temporal trends associated with COVID-19 in-hospital mortality in England: an observational study using administrative data. Lancet Respir Med 9(4):397-406. https://doi.org/10.1016/ S2213-2600(20)30579-8

Onder G, Rezza G, Brusaferro S (2020) Case-fatality rate and characteristics of patients dying in relation to COVID-19 in Italy. JAMA 323(18):1775-1776. https://doi.org/10.1001/jama.2020.4683

Pulakurthi YS, Pederson JM, Saravu K et al (2021) Corticosteroid therapy for COVID-19: A systematic review and meta-analysis of randomized controlled trials. Medicine (Baltimore) 100(20):e25719. https://doi.org/10.1097/MD.0000000000025719

Reese H, Iuliano AD, Patel NN, et al. (2021) Estimated incidence of coronavirus disease (COVID-19) illness and hospitalization-United States, February-September 2020. Clin Infect Dis 72(12):e1010 e1017. https://doi.org/10.1093/cid/ciaa1780

Roth GA, Emmons-Bell S, Alger HM et al (2021) Trends in patient characteristics and COVID-19 in-hospital mortality in the United States during the COVID-19 pandemic. JAMA Netw Open 4(5):e218828. https://doi.org/10.1001/jamanetworkopen.2021.8828

Saito S, Asai Y, Matsunaga N et al (2021) First and second COVID-19 waves in Japan: a comparison of disease severity and characteristics. J Infect 82(4):84-123. https://doi.org/10.1016/j.jinf.2020.10.033

WHO (2020) WHO official update. WHO, Geneva. https://www.euro. who.int/en/health-topics/health-emergencies/coronavirus-covid-19/ news/news/2020/3/who-announces-covid-19-outbreak-a-pandemic. Accessed 1 July 2021

Woolf SH, Chapman DA, Sabo RT, Zimmerman EB (2021)Excess deaths From COVID-19 and other causes in the US, March 1, 2020, to January 2, 2021. JAMA. 325(17):1786-1789. https://doi.org/10. 1001/jama.2021.5199

Publisher's note Springer Nature remains neutral with regard to jurisdictional claims in published maps and institutional affiliations. 


\section{Authors and Affiliations}

\section{Nicola Ughi ${ }^{1}$ (1) Davide Paolo Bernasconi ${ }^{2} \cdot$ Francesca Del Gaudio $^{3}$ - Armanda Dicuonzo ${ }^{3}$ - Alessandro Maloberti ${ }^{4,5}$. Cristina Giannattasio ${ }^{4,5} \cdot$ Paolo Tarsia $^{6} \cdot$ Massimo Puoti $^{5,7}$. Francesco Scaglione ${ }^{8}$. Laura Beltrami $^{9}$. \\ Fabrizio Colombo ${ }^{9}$. Michaela Bertuzzi ${ }^{10}$. Andrea Bellone ${ }^{11}$. Antonella Adinolfi ${ }^{1}$. Maria Grazia Valsecchi ${ }^{2}$. Oscar Massimiliano Epis ${ }^{1}$. Claudio Rossetti ${ }^{3,12}$. on behalf of the Niguarda COVID Working Group}

Nicola Ughi

nicola.ughi@ospedaleniguarda.it

1 Division of Rheumatology, Multispecialist Medical Department, ASST Grande Ospedale Metropolitano Niguarda, Piazza Ospedale Maggiore 3, 20162 Milan, Italy

2 Bicocca Bioinformatics Biostatistics and Bioimaging Centre - B4, School of Medicine and Surgery, University of Milano--Bicocca, Milan, Italy

3 Functional Department for Higher Education, Research, and Development, ASST Grande Ospedale Metropolitano Niguarda, Milan, Italy

4 Division of Cardiology 4, Cardio-Thoraco-Vascular Department, ASST Grande Ospedale Metropolitano Niguarda, Milan, Italy

5 School of Medicine and Surgery, Milano-Bicocca University, Milan, Italy

6 Division of Pneumology, Multispecialist Medical Department, ASST Grande Ospedale Metropolitano Niguarda, Milan, Italy
7 Division of Infectious Disease, Multi-specialist Medical Department, ASST Grande Ospedale Metropolitano Niguarda, Milan, Italy

8 Department of Oncology and Hemato-Oncology, Division of chemical-Clinical and Microbiological Analyses, Department of Laboratory Medicine, ASST Grande Ospedale Metropolitano Niguarda, Università degli Studi di Milano, Milan, Italy

9 Division of Internal Medicine 1, Multispecialist Medical Department, ASST Grande Ospedale Metropolitano Niguarda, Milan, Italy

10 Division of Quality and Clinical Risk, Continuous Quality Improvement, ASST Grande Ospedale Metropolitano Niguarda, Milan, Italy

11 Division of Emergency Medicine and Emergency Room, Department of Emergencies and Admissions (DEA), ASST Grande Ospedale Metropolitano Niguarda, Milan, Italy

12 Functional Department for Higher Education, Research, and Development, Interhospital Functional Department of Nuclear Medicine, ASST Grande Ospedale Metropolitano Niguarda, Milan, Italy 